\title{
PROPERTIES OF THE REGULAR SPECTRUM OF ELEMENTS IN TOPOLOGICAL ALGEBRAS
}

\author{
MATI ABEL
}

\author{
Date of Receiving : $\quad 23.05 .2018$ \\ Date of Revision : 22.08 .2018 \\ Date of Acceptance : 22.08 .2018
}

\begin{abstract}
Relations between the usual spectrum of elements and the regular (or extended) spectrum of elements of topological algebras are described. Conditions when the spectral radius, the regular spectral radius and the radius of boundedness of elements in topological (not necessarily unital and locally convex) algebras coincide are given.
\end{abstract}

Let $A$ be a topological algebra over $\mathbb{C}$ with separately continuous multiplication (in short, a topological algebra). In particular, when the multiplication, as a map $A \times A \rightarrow A$, is continuous, we speak about a topological algebra with jointly continuous multiplication.

Let $\operatorname{Inv} A$ denote the set of all invertible elements in $A$ and Qinv $A$ the set of all quasiinvertible elements in $A$ (that is, of elements $a \in A$, for which there is an element $a_{q}^{-1}$ (the quasi-inverse of $a$ ) such that $a+a_{q}^{-1}=a a_{q}^{-1}=a_{q}^{-1} a$ ). A topological algebra $A$ is called a $Q$-algebra, if the set Qinv $A$ (for unital algebras $\operatorname{Inv} A$ ) is open in $A$. Moreover, a topological algebra $A$ is locally complete (in the locally convex case, G. R. Allan used in [4], p. 401, the term pseudo-complete), if every subalgebra of $A$, generated by a closed, bounded, idempotent and absolutely pseudoconvex subset $U$, is complete in the normed topology (let us remind that $U$ is idempotent, if $U U \subset U$, absolutely $k$-convex, if

$$
U=\Gamma_{k}(U)=\left\{\sum_{v=1}^{n} \alpha_{v} u_{v}: n \in \mathbb{N}, u_{1}, \ldots, u_{n} \in U, \alpha_{1}, \ldots, \alpha_{n} \in \mathbb{C}, \sum_{v=1}^{n}\left|\alpha_{v}\right|^{k} \leqslant 1\right\}
$$

and absolutely pseudoconvex, if $U$ is absolutely $k$-convex for some $k \in(0,1])$. In addition, $A$ is a locally convex (locally $k$-convex or locally $m$-convex) algebra if $A$ has a base of neighbourhoods of zero, consisting of absolutely convex (respectively, absolutely

2010 Mathematics Subject Classification. 46H05, 46H20.

Key words and phrases. Topological algebra, locally convex algebra, regular spectrum of an element, radius of boundedness, idempotently pseudoconvex von Neumann bornology.

Research is in part supported by the institutional research funding IUT20-57 of the Estonian Ministry of Education and Research.

Communicated by: Lourdes Palacios 